\title{
Spin-Wave-Driven Skyrmion Motion in Magnetic Nanostrip
}

\author{
Guangfu Zhang $\mathbb{D},{ }^{1}$ Ye Tian $\mathbb{D}^{1},{ }^{1}$ Yangbao Deng, ${ }^{1}$ Dongchu Jiang, ${ }^{1,2}$ and Shuguang Deng' \\ ${ }^{1}$ School of Communication and Electronic Engineering, Hunan City University, Yiyang 413000, China \\ ${ }^{2}$ Hunan Province Higher Education Key Laboratory of Modeling and Monitoring on the Near-Earth Electromagnetic \\ Environments, Changsha University of Science and Technology, Changsha 410076, China \\ Correspondence should be addressed to Guangfu Zhang; zhanggf@csu.edu.cn
}

Received 25 November 2017; Revised 16 January 2018; Accepted 6 February 2018; Published 1 April 2018

Academic Editor: Paresh Chandra Ray

Copyright (c) 2018 Guangfu Zhang et al. This is an open access article distributed under the Creative Commons Attribution License, which permits unrestricted use, distribution, and reproduction in any medium, provided the original work is properly cited.

\begin{abstract}
The photon-assisted magnetic recording utilizes the ultrafast laser to excite the spin wave in the magnetic nanostructures and accordingly switch its magnetization state. Here, by means of micromagnetic simulation, the motion of magnetic skyrmions, a topologically protected chiral magnet with few nanometer size, induced by the spin wave is studied. It is found that the magnetic skyrmion can move in the same direction of spin-wave propagation, which is first accelerated and then decelerated exponentially. The magnetic skyrmion motion originated from the robust coupling of the spin waves with the skyrmion, through the SW's linear momentum transfer torque acting on the skyrmion. Besides amplitude, the reflectivity of the spin wave by skyrmion has tremendous impact on the velocity of skyrmion motion. The skyrmion velocities are mainly determined by the reflectivity, when the spin-wave amplitude is almost identical. Our results give guidance for the design and development of spin-wave control spintronics.
\end{abstract}

\section{Introduction}

Photon-assisted magnetic recording is a promising direction to expand the capability of the current magnetic storage field represented by the hard disk drive as an indispensable component of modern computers [1]. Typically, this technology utilizes the ultrafast laser to excite the spin wave in the magnetic nanostructures and accordingly switch its magnetization state $[2,3]$. Hence, understanding the interaction between the spin wave and the magnetic nanostructures is an important issue to promote the photon-assisted magnetic recording. Magnetic skyrmions are topologically protected spin textures typical of chiral magnets with few nanometer size, and they are induced by a special kind of exchange interaction known as the Dzyaloshinskii-Moriya interaction (DMI) $[4,5]$. Magnetic skyrmions are appealing as information carriers for potential applications in next-generation spintronic devices, owing to their topological stability, small size, and extremely low energy needed to move it [6-8]. One of the most promising applications of skyrmions is to build racetrack memory (RM). Proposals of manipulations and operations of skyrmions are highly desired. The conventional routes to drive the skyrmions are based on current [4-12]. Although current density needed for their motion is just a few $10^{6} \cdot \mathrm{Am}^{-2}$, the skyrmion velocities under this situation are small $\left(\sim 10^{-4} \cdot \mathrm{ms}^{-1}\right)$. To reach typical domain wall (DW) velocities of $10-100 \mathrm{~ms}^{-1}$, a current density as large as $\sim 10^{11}$ to $10^{12} \cdot \mathrm{Am}^{-2}$ would be necessary, resulting in huge Joule heating.

To overcome the trade-off between current density and the skyrmion velocities, the solution based on the spin Seebeck effect $[13,14]$ and spin wave $[15,16]($ SW) are proposed recent years. Spin waves or magnons also interact with skyrmions and induce magnon-skyrmion scattering [17]. The magnon-skyrmion interaction suggests the possibility of manipulating skyrmions with spin waves. A skyrmion-induced topological magnon Hall effect has been observed [13]. Theoretical studies have demonstrated that the motion of skyrmions could be driven by magnon scattering through the exchange of momenta between the SW and skyrmions [18]. Especially, the driving of skyrmion by SW indicates the possibility of photon-assisted magnetic recording with nanoskyrmions as the record media. In this 
paper, we report the motion of magnetic skyrmions driven by propagating SWs in a nanostrip with perpendicular magnetic anisotropy (PMA) by means of micromagnetic simulations. The SW causes the skyrmion to move in the same direction of spin-wave propagation. The skyrmion motion and their velocity vary remarkably with the frequency and the amplitude of the incident SWs. The skyrmion motions originate from the transfer torque of the SW's linear momentum to the skyrmion, through the partial or complete reflection of the incident SWs from the skyrmion. The skyrmion velocities are mainly determined by the reflectivity, when the SW's amplitude is almost identical.

\section{Simulation Method}

Micromagnetic simulation is an important method to study the dynamic properties of nanomagnets. Its validity has been demonstrated by many experiments. Micromagnetic simulations presented here are performed by the micromagnetic code of the object-oriented micromagnetic framework (OOMMF) [19] including the extension module of the Dzyaloshinskii-Moriya interaction (DMI). The dynamics of magnetization follows the Landau-Lifshitz-Gilbert equation:

$$
\frac{d \vec{m}}{d t}=-|\gamma| \vec{m} \times \vec{H}_{\mathrm{eff}}+\alpha \vec{m} \times \frac{d \vec{m}}{d t}
$$

where $\vec{m}=\vec{M} / M_{S}, \vec{M}$ is local magnetization, and $M_{s}$ is saturation magnetization. $H_{\text {eff }}$ is the total effective field, including Heisenberg exchange, demagnetization, magnetic anisotropy, DMI, and external applied magnetic fields. $\gamma$ is the gyromagnetic ratio. $\alpha$ denotes the damping parameter. For micromagnetic simulations, the finite magnetic nanostrip used here is $1000 \mathrm{~nm}$ long in the $x$ direction, $40 \mathrm{~nm}$ wide in the $y$ direction, and $1 \mathrm{~nm}$ thick in the $z$ direction. The material parameters that were chosen for the simulations correspond to $\mathrm{Co} / \mathrm{Pt}$ multilayers [20]. The values are as follows: saturation magnetization $M_{s}=5.8 \times 10^{5} \cdot \mathrm{A} / \mathrm{m}$, exchange stiffness constant $A=1.5 \times 10^{-11} \mathrm{~J} / \mathrm{m}$, perpendicular magnetic anisotropy (PMA) $K=0.8 \times 10^{6} \cdot \mathrm{J} / \mathrm{m}^{3}$, DMI constant $D=3 \times 10^{-3} \cdot \mathrm{J} / \mathrm{m}^{2}$, and damping coefficient $\alpha=0.015$. The simulated models are discretized into $2 \times 2 \times 1 \mathrm{~nm}^{3}$ cells. The virgin state of the magnetization of the nanostrip is relaxed along the $+z$ direction. A skyrmion is created at $x=200 \mathrm{~nm}$ by the vertical spin-polarized current injection [20] and relaxed to a stable state within a short period of time. To excite the SW, a sinusoidal magnetic field $H=H_{0}$ sin $(2 \pi f t)$ is applied along the $y$-axis at $x \leq 50 \mathrm{~nm}$, where $f$ and $H_{0}$ are the field frequency and amplitude, respectively. The excited SWs are propagated along the long axis of the nanostrip ( $x$-axis), whose frequency equals the frequency of exciting field. In order to prevent reflection of SW s from the right end, we have applied an abrupt absorbing boundary by setting $\alpha=1$ at $x \geq 850 \mathrm{~nm}$.

\section{Results and Discussions}

We first study the motion of the skyrmion driven by monochromatic SWs of different single frequencies within
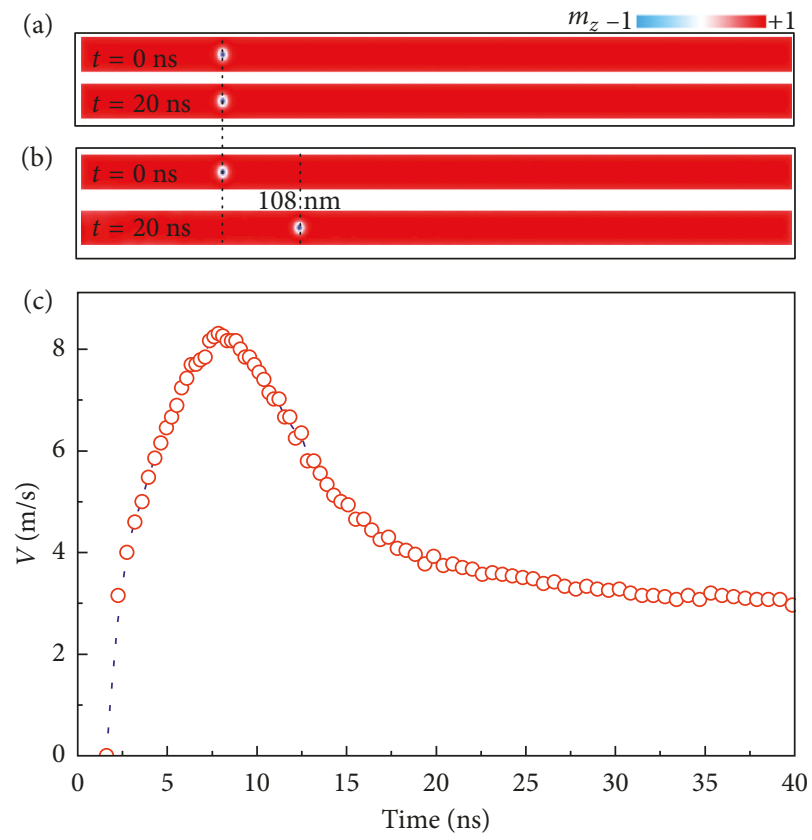

FIgURE 1: The snapshots of the propagation of the skyrmion on the nanostrip and the propagation of the skyrmion driven by the SW injection with the frequencies (a) $40 \mathrm{GHz}$ and (b) $60 \mathrm{GHz}$. (c) The velocity of a skyrmion as a function of time for the SW with frequency $f=60 \mathrm{GHz}$.

the $1 \sim 100 \mathrm{GHz}$ range in increments of $1 \mathrm{GHz}$. The amplitude of exciting field is $H_{0}=50 \mathrm{mT}$. The snapshot images shown in Figures 1(a) and (b) represent the skyrmion motion by incident SWs for different field frequencies at different times. For the SW with frequency $f=40 \mathrm{GHz}$, the SW cannot move the magnetic skyrmion even, and the skyrmion is well stable and intact due to the topological protection. However, for the SW with frequency $f=60 \mathrm{GHz}$, there is a relatively large skyrmion motion in the same direction of the SW propagation, and the skyrmion remains intact during its interactions with SWs. Figure 1(c) shows the skyrmion's velocity as a function of time for the SW with frequency $f=60 \mathrm{GHz}$. At first, the skyrmion moves with an acceleration stage, and then, the motion velocity decreases. A skyrmion is at rest initially. When the SW passes through the skyrmion, robust interactions between the incident SW and the skyrmion induce a reaction torque on the skyrmion, making the skyrmions move. At the acceleration stage, the SW is blocked by skyrmions. The transfer torque from SW to skyrmions is increased, leading to increase in the skyrmion velocity. After a long enough time, the skyrmion moves gradually away from the exciting region, and the velocity also decays since the SW decays.

The average velocity $V$ of 20 nanoseconds versus the frequency $f$ is shown in Figure 2(a), which displays oscillation behavior. There are four obvious bulges in the curve, and the scopes of the corresponding frequency are 56$60 \mathrm{GHz}, \quad 60-64 \mathrm{GHz}, \quad 70-79 \mathrm{GHz}$, and $85-100 \mathrm{GHz}$, respectively. The maximum velocity appears at $f=58 \mathrm{GHz}$. For $f<56 \mathrm{GHz}$ and $80 \mathrm{GHz} \leq f \leq 84 \mathrm{GHz}$, the SW leads to no motion or a small extent of motion. Similar to dynamics 


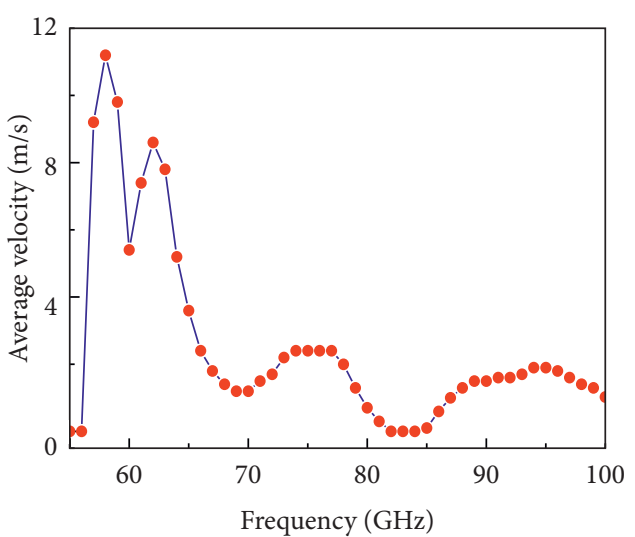

(a)

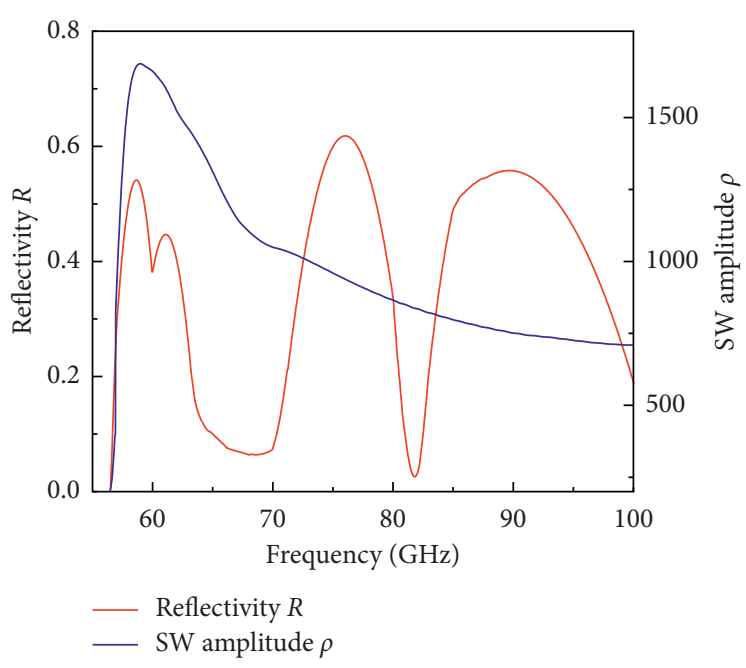

(b)

Figure 2: (a) The average velocity $V$ of 20 nanoseconds versus the frequency $f$; (b) the reflection coefficients $R$ of the SW passing through the skyrmion (blue solid line) and the amplitude of the SW $\rho$ (red solid line) as a function of frequency.

of SW-indued wall motion [21-23], skyrmion dynamics motion can be understood. The propagation of the SW produces magnonic spin current. In case a magnon is reflected by the skyrmion, its spin is kept constant, but its momentum is changed by $2 h k$. Here, $k$ is the wave vector of the SW. The transfer of momentum between the magnon and the skyrmion also induces torque acting on the skyrmion and pushes it to move. The skyrmion's velocities are determined by the reflected magnons, which depend on the magnon density $n$ and the reflection coefficients $R$. $n$ is directly proportional to the square of the spin-wave amplitude [23].

In order to elucidate the underlying physics of the observed frequency-dependent skyrmion motions, we performed additional micromagnetic simulations. A sinefunction field $H=H_{0} \sin \left(2 \pi f_{c} t\right) /\left(2 \pi f_{c} t\right) \hat{y}$ with $H_{0}=3 \mathrm{mT}$ and $f_{c}=100 \mathrm{GHz}$ is used at the same nanostrip with and without a single skyrmion. The SWs of frequency $f$ from 0 to $100 \mathrm{GHz}$ can be excited. The SW amplitudes were calculated by the fast Fourier transformation of the temporal evolutions of the magnetization for the nanostrip. The reflection coefficients $R$ are $1-\rho_{\text {sk }} / \rho_{0}$, where $\rho_{\text {sk }}$ and $\rho_{0}$ are the SW average amplitudes at $x=225-255 \mathrm{~nm}$ for the case with $\left(\rho_{\text {sk }}\right)$ and without $\left(\rho_{0}\right)$ the skyrmion. Figure 2(b) shows the frequency dependencies of the spin-wave average amplitude $\rho$ at $x=145-175 \mathrm{~nm}$ for the case without skyrmion and the reflection coefficients $R$ by the skyrmion. The SW having frequencies lower than $56 \mathrm{GHz}$ are prohibited to propagate in the nanostrip. It can be seen from Figure 2(b) that the amplitude $\rho$ is almost zero in the region of frequencies lower than $56 \mathrm{GHz}$. For frequencies larger than $56 \mathrm{GHz}$, the amplitude $\rho$ first increases with the increase in frequency, and a maximum value appears at $f \approx 59 \mathrm{GHz}$. And then, the average amplitude $\rho$ decreases with the increase in frequency. The reflection coefficients $R$ are also sensitively dependent on the frequency. The reflectivity in the ranges $f=65-69 \mathrm{GHz}$ and $f=81-83 \mathrm{GHz}$ is extremely small, as low as 0.1 , meaning that the SWs pass through the skyrmion almost without reflection. For other frequencies in the range of $56-100 \mathrm{GHz}$, the SWs are partially reflected, and they display the resonance reflective phenomenon. The reflective behavior results from the stray field due to the transversely confined dimension. Also, the reflectivity is related to the inherent oscillation modes of the skyrmion. We calculated the SW frequency spectrum of the nanostrip with a single skyrmion. From the spatial distributions of the FFT powers of different spin-wave resonance peaks, the breathing and the clockwise gyrotropic SW modes of the skyrmion are found in the frequencies $58 \mathrm{GHz}$ and $62.25 \mathrm{GHz}$, respectively. There are some mixed modes between the breathing mode and quantized SW eigenmodes in the ranges $66-77 \mathrm{GHz}$ and $87-95 \mathrm{GHz}$. These are displayed on our $R(f)$ curve as well, where four obvious bulges are corresponding to these normal oscillation modes of the skyrmion.

By comparing oscillation behavior of the reflective and average velocity $(V)$ versus the frequency $f$, one can realize that the resonant ranges are in good agreement. This reflects the fact that the frequency-sensitive skyrmion motion is closely related to the reflection coefficients $R$. For $f<56 \mathrm{GHz}$, the SW cannot drive the skyrmion to move since it forbids propagation in the nanostrip. The incident SWs of the specific frequency range $f=56-70 \mathrm{GHz}$ allow for a relatively large skyrmion motion in the same direction of the SW propagation because of larger amplitude of the SW. It is worth noting that there is clearly a drop of transmission in the $V(f)$ curve as well, where at frequency of about $60 \mathrm{GHz}$. A drop appears in our $R(f)$ curve as well at the same frequency, corresponding to a relatively smaller reflectivity. However, the SW's amplitude decreases with the increase of frequency. Thus, the skyrmion velocity is mainly determined by the reflectivity, when the SW's amplitude is almost identical. This has the profound evidence that there is relatively large skyrmion motion velocity induced by the SW of specific frequency ranges $f=70-82 \mathrm{GHz}$ and $f=85-100 \mathrm{GHz}$, in which 
SWs have a small amplitude but a considerable reflection from the skyrmion. Contrastingly, for $82 \mathrm{GHz} \leq f \leq 84 \mathrm{GHz}$, the SW allows passing through the skyrmion and has a very weak reflection $(R<0.09)$. Furthermore, the amplitude is extremely small. Low reflectivity and amplitude of the incident SW all cause a small dragging torque to act on the skyrmion. The skyrmion has a small extent of motion, even without moving. We also notice that the spin wave drives the wall to move in the opposite direction of the incident spin wave reported in [23]. However, the backward skyrmion motion similar to the spin-wave-driven domain wall motions is unable to observe. The reason may be that the spin-wave amplitude is too small.

\section{Conclusions}

In summary, we have studied the skyrmion motion in a PMA nanostrip induced by SW injection using micromagnetic simulations. It is found that the skyrmion motion is strongly dependent on the SW's propagating characteristics passing through the skyrmion. For the given dimensions of the PMA nanostrip, the skyrmion is not driven by SWs with a lower frequency than $56 \mathrm{GHz}$ because they are prohibited from propagation in the nanostrip. For frequencies larger than $56 \mathrm{GHz}$, the SWs partially transmit through the skyrmion, and the reflection coefficients $R$ display oscillation behavior with the increase in frequency. The velocity of a skyrmion first increases and then decreases under the monochromatic SWs. The average velocity $(V)$ of 20 nanoseconds is strongly dependent on the frequency and amplitude of the incident SWs. The larger amplitude of the SW leads to a larger average velocity of the skyrmion. The skyrmion velocity is mainly determined by the reflectivity, when the SW's amplitude is almost identical. This work would also serve as an indispensable guide to scientists and future applications to skyrmionics driven by the SWs.

\section{Conflicts of Interest}

The authors declare that there are no conflicts of interest.

\section{Acknowledgments}

This work was supported by the National Natural Science Foundation of China (Project nos. 11604091, 1547186, and 61471164) and the Research Foundation of Education Bureau of Hunan Province, China (Project no. 16B048). Dong-chu Jiang acknowledges the support of the Open Research Fund of the Hunan Province Higher Education Key Laboratory of Modeling and Monitoring on the Near-Earth Electromagnetic Environments (no. 2), Changsha University of Science and Technology.

\section{References}

[1] A. Stupakiewicz, K. Szerenos, D. Afanasiev, A. Kirilyuk, and A. V. Kimel, "Ultrafast nonthermal photo-magnetic recording in a transparent medium," Nature, vol. 542, no. 7369, pp. 71-74, 2017.
[2] A. Kamimaki, S. Iihama, Y. Sasaki, Y. Ando, and S. Mizukami, "Micro-focused pulse laser-induced propagating spin waves in permalloy films with different thicknesses," IEEE Transactions on Magnetics, vol. 14, no. 8, pp. 1-4, 2015.

[3] S.-J. Yun, C.-G. Cho, and S.-B. Choe, "Simultaneous excitation of two different spinwave modes by optical ultrafast demagnetization," Applied Physics Express, vol. 8, no. 6, Article ID 063009, 3 pages, 2015.

[4] S. Woo, K. Litzius, B. Krüger et al., "Observation of roomtemperature magnetic skyrmions and their current-driven dynamics in ultrathin metallic ferromagnets," Nature materials, vol. 15, no. 5, pp. 501-506, 2016.

[5] S. Mhlbauer, B. Binz, F. Jonietz et al., "Skyrmion lattice in a chiral magnet," Science, vol. 323, no. 5916, pp. 915-919, 2009.

[6] R Tomasello, E Martinez, R Zivieri et al., "A strategy for the design of skyrmion racetrack memories," Scientific Reports, vol. 4, no. 1, Article ID 06784, 7 pages, 2014.

[7] X. Zhang, M. Ezawa, and Y. Zhou, "Magnetic skyrmion logic gates: conversion, duplication and merging of skyrmions," Scientific Reports, vol. 5, no. 1, Article ID 09400, 8 pages, 2015.

[8] Y. Zhou and M. Ezawa, "A reversible conversion between a skyrmion and a domain-wall pair in a junction geometry," Nature Communications, vol. 5, no. 6, Article ID 5652, 8 pages, 2014.

[9] I. Purnama, W. L. Gan, D. W. Wong, and W. S. Lew, "Guided current-induced skyrmion motion in 1D potential well," Scientific Reports, vol. 5, no. 1, Article ID 10620, 9 pages, 2015.

[10] L. Dong, J. P. Degrave, M. J. Stolt, Y. Tokura, and S. Jin, "Current-driven dynamics of skyrmions stabilized in $\mathrm{MnSi}$ nanowires revealed by topological Hall effect," Nature Communications, vol. 6, no. 1, Article ID 8217, 8 pages, 2015.

[11] A Fert, V Cros, and J Sampaio, "Skyrmions on the track," Nature Nanotechnology, vol. 8, no. 3, pp. 152-156, 2013.

[12] J. Iwasaki, M. Mochizuki, and N. Nagaosa, "Current-induced skyrmion dynamics in constricted geometries," Nature Nanotechnology, vol. 8, no. 10, pp. 742-747, 2013.

[13] M. Mochizuki, X. Z. Yu, S. Seki et al., "Thermally driven ratchet motion of a skyrmion microcrystal and topological magnon Hall effect," Nature Materials, vol. 13, no. 3, pp. 241-246, 2014.

[14] S. Z. Lin, C. D. Batista, C. Reichhardt, and A. Saxena, "AC current generation in chiral magnetic insulators and skyrmion motion induced by the spin Seebeck effect," Physical Review Letters, vol. 112, no. 18, Article ID 187203, 5 pages, 2014.

[15] J. Ding, X. Yang, and Tao Zhu, "The motion of magnetic skyrmions driven by propagating spin waves," IEEE Transactions on Magnetics, vol. 51, no. 11, Article ID 1500504, 4 pages, 2015.

[16] X. Zhang, M. Ezawa, D. Xiao, G. P. Zhao, Y. Liu, and Y. Zhou, "All-magnetic control of skyrmions in nanowires by a spin wave," Nanotechnology, vol. 26, no. 22, Article ID 225701, 8 pages, 2015.

[17] J. Iwasaki, A. J. Beekman, and N. Nagaosa, "Theory of magnon-skyrmion scattering in chiral magnets," Physical Review B, vol. 89, no. 6, Article ID 064412, 7 pages, 2014.

[18] S. Schroeter and M. Garst, "Scattering of high-energy magnons off a magnetic skyrmion," Low Temperature Physics, vol. 41, no. 10, pp. 817-825, 2015.

[19] M. J. Donahue and D. G. Porter, "The Object Oriented MicroMagnetic Framework (OOMMF) Project at ITL/NIST," 2017, http://math.nist.gov/oommf/.

[20] J Sampaio, V Cros, S Rohart, A. Thiaville, and A. Fert, "Nucleation, stability and current-induced motion of isolated 
magnetic skyrmions in nanostructures," Nature Nanotechnology, vol. 8, no. 11, pp. 839-844, 2013.

[21] J. Yang, M. W. Yoo, and S. K. Kim, "Spin-wave-driven highspeed domain-wall motions in soft magnetic nanotubes," Journal of Applied Physics, vol. 118, no. 16, Article ID 163902, 5 pages, 2015.

[22] P. Yan, X. S. Wang, and X. R. Wang, "All-magnonic spintransfer torque and domain wall propagation," Physical Review Letters, vol. 107, no. 17, Article ID 163902, 5 pages, 2011.

[23] X.-G. Wang, G.-H. Guo, Y.-Z. Nie, G.-F. Zhang, and Z.-X. Li, "Domain wall motion induced by the magnonic spin current," Physical Review B, vol. 86, no. 5, Article ID 054445, 5 pages, 2012. 


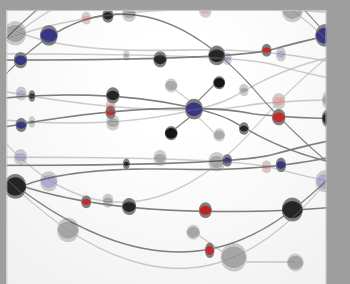

The Scientific World Journal
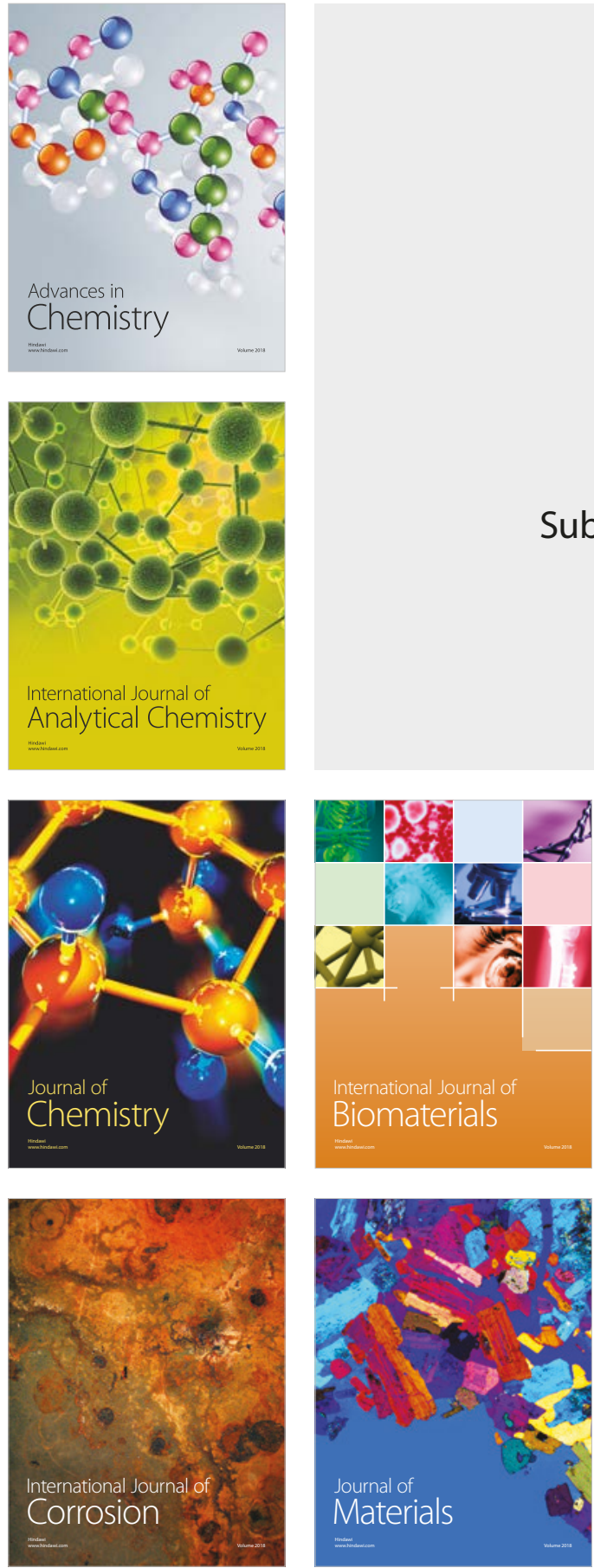

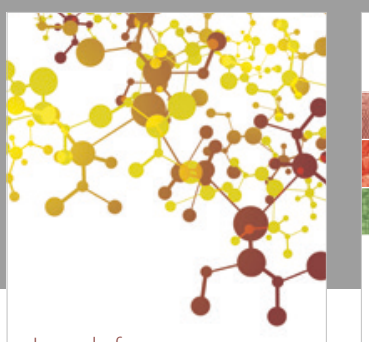

Journal of

Applied Chemistry
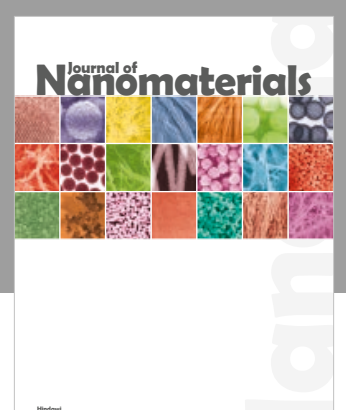

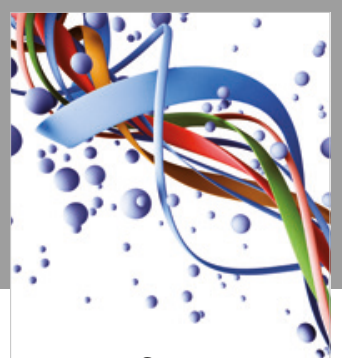

Scientifica

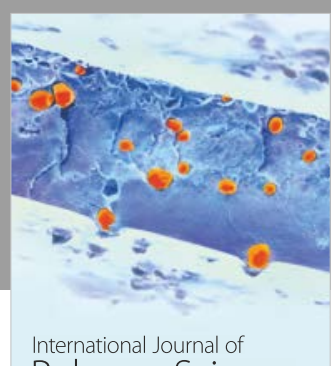

Polymer Science

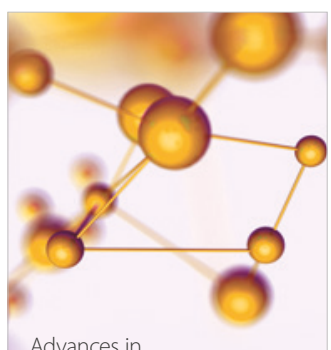

Physical Chemistry
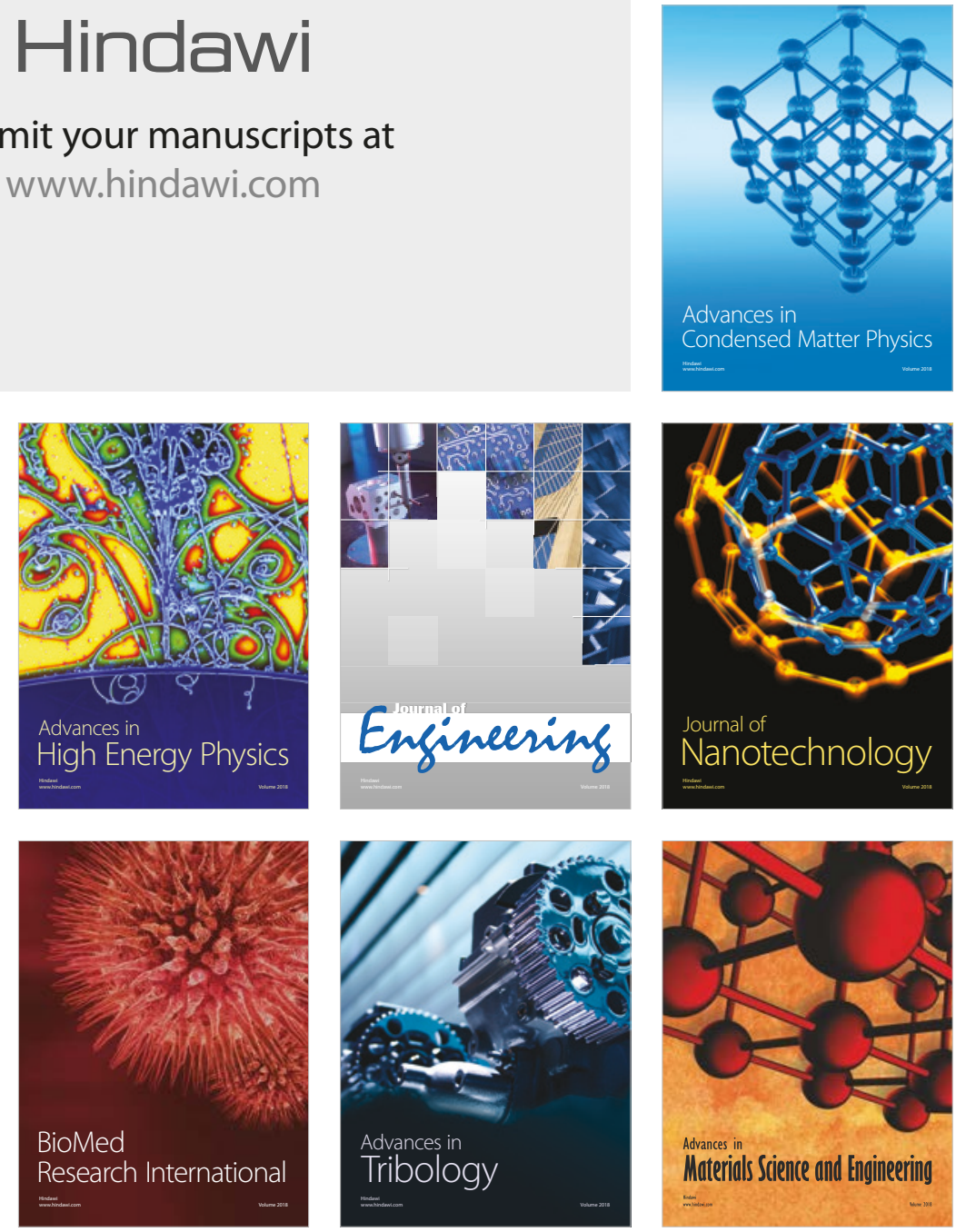